\title{
Atividade física como fator protetor para o extremo baixo peso em idosos assistidos por uma operadora de saúde
}

\section{Physical activity as a protective factor for extreme low weight in elderly assisted by a health care operator}

Deborah Lorenzo Gomez Silva ${ }^{1}$, DRafaela Ramos D’Oliveira ${ }^{1}$, Beatriz Myung Jin Kim ${ }^{1}$, Ana Flávia de Lima Santos ${ }^{1}$, Eduardo Antonio Barros Silva Junior ${ }^{1}$, (D) Henrique Souza Barros de Oliveira ${ }^{1}$, (DMaria Elisa Gonzalez Manso ${ }^{1,2}$

\section{RESUMO}

Objetivo: Investigar a influência de fatores relacionados aos aspectos multidimensionais do envelhecimento em um grupo de idosos com baixo peso assistidos pelo setor privado de saúde. Métodos: Estudo transversal conduzido com 243 idosos com índice de massa corpórea <23 $\mathrm{kg} / \mathrm{m}^{2}$, todos assistidos por uma operadora de planos de saúde, no município de São Paulo, SP, Brasil, alocados em dois grupos: baixo peso e extremo baixo peso. Para a coleta dos dados empregou-se inquérito contendo informações relacionadas aos aspectos multidimensionais do envelhecimento. Os dados obtidos foram submetidos a análise descritiva das variáveis, com posterior análise comparativa através dos testes t Student e Qui-Quadrado ou exato de Fisher, quando necessário. Para variáveis com significância estatística, aplicou-se regressão logística e calculou-se as Odds Ratio, com intervalo de confiança de 95\%. Resultados: Observou-se que, a idade avançada $(p=0,044)$, dificuldade de mobilidade $(p=0,011)$ e baixos níveis de atividade física $(p=0.021)$ influenciam significativamente os idosos em estado nutricional de extremo baixo peso. Por meio do modelo de regressão logística, verificou-se que para cada uma hora de atividade física praticada pelos idosos deste grupo, os riscos de evolução para extremo baixo peso diminuem em até $14 \%$. Conclusão: A pratica regular e orientada de atividade física praticada por idosos com algum grau de déficit nutricional é capaz de prevenir e/ou retardar desfechos indesejados em grupos susceptíveis

Palavras-chave: Serviços de Saúde para Idosos, Estado Nutricional, Atividade Motora, Estilo de Vida

\footnotetext{
${ }^{1}$ Centro Universitário São Camilo

${ }^{2}$ Pontifícia Universidade Católica de São Paulo PUC-SP
}

\section{Correspondência}

Henrique Souza Barros de Oliveira

E-mail: heeenry.barros@icloud.com

Recebido: 12 Agosto 2020

Aceito: 03 Junho 2020

\section{Como citar}

Silva DLG, D'Oliveira RR, Kim BMJ, Santos AFL, Silva Junior EAB, Oliveira HSB, et al. Atividade física como fator protetor para o extremo baixo peso em idosos assistidos por uma operadora de saúde. Acta Fisiatr. 2020;27(1):41-44

DOI: 10.11606/issn.2317-0190.v27i1a172965

\begin{abstract}
Objective: Investigate the influence of factors related to multidimensional aspects of aging in a group of elderly assisted by the private health sector. Methods: Cross-sectional study conducted with 243 elders with a body mass index $<23 \mathrm{~kg} / \mathrm{m}^{2}$ assisted by a health plan in the city of São Paulo, SP, Brazil, with them being allocated into two groups: low weight and extreme low weight. For data collection, a survey developed by the researchers themselves containing information related to multidimensional aspects of aging was employed. The obtained data were submitted to a descriptive analysis of the variables and a subsequent comparative analysis through Student's t-distribution, Chi-squared or Fisher's exact tests, when necessary. For variables with statistical significance, logistic regression was applied and the odds ratio, with a 95\% confidence interval, was calculated. Results: It was observed that advanced age $(p=0.044)$, mobility difficulty $(p=0.011)$ and low levels of physical activity $(p=0.021)$ significantly influence the elderly in extremely low weight nutritional status. Moreover, through the logistic regression model, it was found that, for this group, every hour of physical activity practiced by underweight elders, the risks of evolution to an extreme low weight status decrease by up to $14 \%$. Conclusion: The regular and oriented practice of physical activity by the elderly with some degree of nutritional deficit is able to prevent and/or delay unwanted outcomes in susceptible groups.
\end{abstract}

Keywords: Health Services for the Aged, Nutritional Status, Motor Activity, Life Style 


\section{INTRODUÇÃO}

O envelhecimento humano é caracterizado como um processo sequencial, individual, acumulativo, irreversível, universal, não patológico, próprio a todos os membros de uma espécie e identificado por alterações fisiológicas capazes de modificar os principais sistemas associados às necessidades nutricionais do idoso. ${ }^{1,2}$ Estas mudanças podem tornar o idoso propenso a perdas que ocorrem tanto na capacidade de adaptação ao meio ambiente quanto na funcionalidade, contribuindo para modificações na forma de se alimentar, as quais causam comprometimento do estado nutricional e agravam as perdas mencionadas, em um ciclo viciosa que afeta a saúde das pessoas que compõem este segmento populacional. ${ }^{2}$

Neste contexto, a desnutrição é o mais importante distúrbio nutricional relacionado ao envelhecimento, associando-se ao aumento dos índices de mortalidade e morbidade, à maior susceptibilidade às infecções, à redução da capacidade funcional e cognitiva e à diminuição dos níveis de qualidade de vida., ${ }^{3,4}$ A detecção e o manejo precoce deste tipo de condição podem contribuir para prevenção de inúmeros agravos específicos à saúde do idoso, tais como: síndrome da fragilidade, síndrome da imobilidade; síndrome demencial; sarcopenia; síndrome consumptiva, dentre outras afecções. ${ }^{4}$

Considera-se como indicadores de baixo peso no idoso os seguintes parâmetros: (i) perda de peso involuntária de aproximadamente $5 \%$ em um mês, $7,5 \%$ em três meses e $10 \%$ em seis meses; (ii) peso baixo para a altura, inferior a mais de $20 \%$ do peso corporal ideal; (iii) Índice de Massa Corporal (IMC) $<23 \mathrm{~kg} /$ $\mathrm{m}^{2}$; (iv) albumina sérica $<3,5 \mathrm{mg} / \mathrm{dL}$; (v) nível de colesterol sérico total $<160 \mathrm{mg} / \mathrm{dL}$; (vi) mudanças na funcionalidade; (vii) alimentação inadequada; (viii) circunferência muscular do braço inferior ao percentil 10; (ix) prega cutânea tricipital menor que o percentil $10 .^{4}$

De maneira geral, as pesquisas utilizam - IMC como ferramenta para determinar e avaliar o estado nutricional do indivíduo e para investigar a associação entre este estado com o risco de morbidade, mortalidade, fatores agravantes e protetores. ${ }^{5}$ Entretanto, há divergências na forma de estratificar a população idosa quanto ao IMC. Assim, a Organização Pan-Americana de Saúde (OPAS), considera um indivíduo como estando com baixo peso quando seu IMC for $\leq 23 \mathrm{~kg} / \mathrm{m}^{2}$, eutrófico quando IMC estiver entre 24 a 27 $\mathrm{kg} / \mathrm{m}^{2}$, com sobrepeso ou pré-obeso para IMC entre 28 a $29 \mathrm{~kg} / \mathrm{m}^{2}$ e obeso quando o IMC for $\geq 30 \mathrm{~kg} / \mathrm{m}^{2}$. 5,6

A World Health Organization (WHO), por sua vez, utiliza pontos de corte distintos dos apresentados anteriormente, considerando um indivíduo com baixo peso quando seu IMC for $<18,5 \mathrm{~kg} / \mathrm{m}^{2}$, eutrófico quando IMC estiver entre 18,5 a $24,99 \mathrm{~kg} / \mathrm{m}^{2}$, sobrepeso quando IMC se encontrar entre 25 a $29,99 \mathrm{~kg} / \mathrm{m}^{2}$ e obeso quando IMC $\geq 30 \mathrm{~kg} / \mathrm{m}^{2}$. 6,7 Já a Nutrition Screening Initiative (NSI), criada a partir do conjunto de três organizações internacionais: American Academy of Family Physicians, American Dietetic Association e National Council on the Aging Inc., adota como ponto de corte, a fim de considerar uma pessoa idosa como tendo baixo peso, o IMC $<22 \mathrm{~kg} /$ $\mathrm{m}^{2}$.6,8

Entendendo que a investigação dos fatores associados a situação nutricional de idosos é de suma relevância e pode subsidiar ajustes nas políticas de prevenção de agravos e a promoção à saúde, prioridade dada pelo governo brasileiro ao setor de saúde suplementar, ${ }^{9}$ pensou-se nesta pesquisa. Ressalva-se que há uma lacuna na literatura, não existindo pesquisas que explorem a influência de baixo peso na população geriátrica do setor privado de saúde brasileiro.

\section{OBJETIVO}

Determinar a influência de fatores relacionados aos aspectos multidimensionais do envelhecimento em um grupo de idosos com baixo peso assistidos por uma operadora de planos de saúde, no município de São Paulo, SP, Brasil.

\section{MÉTODOS}

Estudo transversal de base populacional que investigou um grupo de 243 idosos classificados como tendo baixo peso, assistidos por uma Operadora de Planos de Saúde (OPS), localizada no município de São Paulo - SP, Brasil, durante o ano de 2018.

Dentre os indivíduos que são assistidos por esta operadora, foram selecionados para compor o grupo pesquisado apenas os idosos com idade igual ou superior a 60 anos, de ambos os sexos, não institucionalizados, com Índice de Massa Corpórea (IMC) corrigido para a idade $<23 \mathrm{~kg} / \mathrm{m}^{2}$. 2,5,6 Os indivíduos que possuíam condições médicas que impossibilitasse a participação na pesquisa foram excluídos.
Inicialmente, todos os idosos participantes foram contatados por telefone, tomando ciência do ingresso opcional, do sigilo dos seus dados e que a sua não aceitação não interferiria na atenção prestada pela OPS. Somente após todos assinarem o termo de consentimento livre e esclarecido, uma equipe de enfermeiros treinados e capacitados coletou os dados nas residências de cada participante.

Para a coleta dos dados empregou-se um questionário contendo informações sociodemográficas, tais como: idade; sexo; situação conjugal; cidade em que reside; presença de morbidades crônicas em tratamento; uso de medicamentos; pratica de atividade física em uma semana usual e hábito do consumo de tabaco. Foram aplicados ainda os índices de dependência para as Atividades Básicas de Vida Diária - ABVD (Katz Índex $)^{10}$ e de dependência para as Atividades Instrumentais de Vida Diária - AIVD (Lawton Índex), ${ }^{10}$ assim como foi realizado o teste de Time Get Up and Go (TUG) para verificação da mobilidade e o risco de quedas. ${ }^{11}$

Para avaliação do estado nutricional, utilizou-se a ferramenta Mini-Avaliação Nutricional MAN em versão reduzida, que avalia o risco nutricional a partir da análise da diminuição e qualidade da ingesta alimentar, perda de peso, mobilidade, aspectos psicológicos e índice de massa corporal. Este instrumento identifica indivíduos idosos em risco de desnutrição ou sobrepeso, sendo método simples, rápido de ser aplicado e validado. ${ }^{2}$ Para analisar a ingestão de substancias alcoólicas, empregou-se o Teste de Identificação de Distúrbio de Uso do Álcool (AUDIT). ${ }^{12}$

Quanto as medidas antropométricas, utilizou-se um estadiômetro portátil com trena retrátil, de extensão máxima de 200 $\mathrm{cm}$ e de precisão de $0,1 \mathrm{~cm}$ para altura e uma balança eletrônica portátil, com capacidade máxima de $150 \mathrm{~kg}$ e graduação de $100 \mathrm{~kg}$ para mensuração do peso.

A classificação do estado nutricional deste estudo adotou os parâmetros da Organização Pan-Americana de Saúde (OPAS), ${ }^{5}$ que leva em consideração as modificações na composição corporal próprias do envelhecimento. Em relação aos idosos com baixo peso selecionados, categorizou-se dois grupos quanto a severidade do estado nutricional para realização de análises comparativas: (1) baixo peso - IMC 23 a $21 \mathrm{~kg} / \mathrm{m}^{2}$ e (2) extremo baixo peso - IMC $\geq 20 \mathrm{~kg} / \mathrm{m}^{2}$.

Para análise descritiva, empregouse medidas de distribuição de frequência absoluta e relativa para variáveis categóricas, 
enquanto que medidas de tendência central e de dispersão foram empregadas para variáveis contínuas. Para análise comparativa entre os grupos de baixo peso e as variáveis especificadas acima, aplicou-se os testes $\mathrm{t}$ Student e Qui-Quadrado ou exato de Fisher (F), quando necessário, para variáveis quantitativas e qualitativas, respectivamente.

As variáveis que apresentaram significância estatística nas análises anteriormente citadas, foram selecionadas para compor o modelo de regressão logística através do método de Stepwise Forward para identificação dos fatores relacionados aos idosos com baixo peso. Para análise de regressão logística foram calculadas as respectivas Odds Ratio (OR) e o intervalo de confiança de $95 \%$ das variáveis inseridas neste modelo. Os dados foram analisados no software Statistical Package for Social Sciences (SPSS ${ }^{\circ}$ ) for Windows e considerou-se um nível de significância estatística de $5 \%$ para todos os testes.

Este estudo foi conduzido dentro dos preceitos éticos recomendados pela Resolução CNS 466-2012, tendo sido aprovado pelo Comité de Ética em Pesquisa da Pontifícia Universidade Católica de São Paulo, CAAE 87307118.5.0000.5482, Número do Parecer: 2.626.054.

\section{RESULTADOS}

O grupo estudado apresentou uma média de idade igual a 78,5 anos (DP 8,1), sendo composto, em sua maioria, por pessoas do sexo feminino $(65,8 \%, n=160)$. Quanto ao estado conjugal, a maioria destes idosos ou são casados ou vivem em situação de união estável $(48,1 \%, n=117)$, mas $42 \%(n=102)$ são viúvos.

Em relação a saúde, todos apresentavam, pelo menos, uma doença crônica degenerativa, sendo as mais frequentes: hipertensão arterial sistêmica $(68,7 \%, \mathrm{n}=167)$, diabetes mellitus tipo II não-insulino-dependente $(22 \%, n=55)$ e cardiomiopatia isquêmica $(1,2 \%, n=3)$. A média de comorbidades deste grupo foi igual a 6,2 (DP 2,7), havendo indivíduos portadores de até 15 doenças concomitantes. Os idosos em tratamento medicamentoso faziam uso médio de 6,7 (DP 3,9) fármacos/idoso, variando entre 21 e zero fármacos/dia.

Encontrou-se, neste grupo de idosos com baixo peso, predomínio de hábito alimentar considerado inadequado, hábito este avaliado pelas questões $\mathrm{J}$ até $\mathrm{M}$ do instrumento MAN $(58 \%, n=141)$. A maioria dos idosos que compõem o grupo pesquisado não apresentou risco para dependência alcoólica pelo AUDIT
$(100 \%, n=243)$ e nem mencionaram o habito de fumar $(92 \%, n=225)$. A pratica de atividade física foi verificada em uma média geral de 1,25 (DP 2,28) idosos com baixo peso.

Quanto ao grau de dependência, observou-se que apenas $8,2 \%(n=20)$ e $29,2 \%$ $(n=71)$ dos idosos participantes da pesquisa eram dependentes em algum grau para as ABVD e AIVD, respectivamente. Em relação à avaliação pelo teste de TUG, notou-se que $59,7 \%(n=145)$ destes idosos não apresentam nenhum grau de dificuldade.

De acordo com os parâmetros do IMC corrigidos para a idade, o estado nutricional deste grupo atingiu uma média de $21 \mathrm{~kg} / \mathrm{m}^{2}$ (DP 1,6), variando entre 16 e $23 \mathrm{~kg} / \mathrm{m}^{2}$. Entre os idosos pesquisados, encontrou-se $70 \%$ ( $n$ $=170) \mathrm{com}$ baixo peso e $30 \%(n=73) \mathrm{com}$ extremo baixo peso, quando classificados conforme os índices anteriormente especificados. A análise comparativa entre estes dois grupos constatou influência significativa entre idade avançada $(p=0,044)$, baixo nível de atividade física $(p=0,021)$ e dificuldade em algum grau de mobilidade ( $p$ $=0,011$ ) em idosos classificados como tendo extremo baixo peso.

Avaliou-se ainda as OR das variáveis desse modelo, encontrando-se que a cada uma hora a mais de atividade física praticada por idosos com baixo peso, as chances de evolução para extremo baixo peso diminui em $14 \%(O R=0,86 ;$ IC $95 \%=0,74-0,99 ; p$ $=0,048)$.

\section{DISCUSSÃO}

A prevalência de idosos com idade avançada neste grupo pesquisado encontrase em consonância com o perfil demográfico encontrado para idosos vinculados ao setor privado de saúde brasileira, onde se constata que estes possuem idades mais avançadas que a população idosa no geral. Observa-se ainda neste grupo o predomínio de mulheres, em união estável e viúvas, como também destacam outras pesquisas realizadas em idosos do setor suplementar. ${ }^{13,14}$

O perfil de doenças encontrado para este grupo também repete o padrão não só da população vinculada a planos de saúde no Brasil, como da população idosa como um todo no país, onde a hipertensão arterial é a afeç̧ão crônica mais prevalente e a presença de múltiplas morbidades em associação também é frequente. Não obstante, o número de doenças em associação encontrado para este grupo de idosos mostrou-se mais elevado do que para a população em geral. ${ }^{15}$
Este número elevado de doenças, associado à alimentação inadequada, baixos níveis de atividade física e comprometimento do estado nutricional (baixo peso) encontrado para este grupo, permite a inferência de que se trata de um grupo com alto risco tanto para a ocorrência de desfechos adversos à saúde quanto para mortalidade.

Além do citado, estes idosos pesquisados apresentam uma média geral de consumo diário de medicamentos/idosos superior ao encontrado na literatura nacional para idosos do mesmo setor de assistência à saúde. ${ }^{16,17} \mathrm{~A}$ elevada utilização de fármacos em associação eleva o risco de desencadeamento de eventos adversos, tais como: intoxicação, prescrição em cascata e a tríade iatrogênica (definida como consumo de pelo menos um medicamento potencialmente inapropriado associado ao uso contínuo de polifarmácia e a presença de potenciais interações medicamentos e reações adversas). ${ }^{16,17} \mathrm{O}$ uso de múltiplos fármacos também pode interferir tanto na absorção de nutrientes quanto na ingesta de alimentos, podendo piorar o estado nutricional dos idosos. ${ }^{2}$

Sabe-se também da relevância do estilo de vida e da capacidade funcional para manter o equilíbrio do estado nutricional de indivíduos longevos. ${ }^{2} \mathrm{O}$ estilo de vida pode ser caracterizado como uma associação de hábitos e costumes vivenciados pelos indivíduos durante a vida, sendo a alimentação, tabagismo, consumo de substancias alcoólicas e a pratica de atividade física alguns destes. ${ }^{18}$ Neste grupo de idosos pesquisados, que já apresentam baixo peso, encontrou-se maioria com hábitos alimentares inadequados e baixos níveis de atividade física.

Verificou-se ainda que, além da idade avançada, os baixos níveis de atividade física e a dificuldade de mobilidade influenciaram de maneira significativa o estado nutricional dos idosos classificados como tendo extremo baixo peso. A literatura destaca que, quanto mais avançada a idade, maior é a perda de massa muscular, em virtude da maior tendência à proteólise muscular e da desregulação do anabolismo proteico no idoso, principalmente quando este permanece em repouso. Ademais, a idade avançada, se associada à baixos níveis de atividade física, é considerada ainda fator de risco importante para o desenvolvimento de quadro sarcopênico em idosos. A junção de fatores críticos acentua a perda de massa muscular e, consequentemente, massa corporal total em indivíduos idosos. ${ }^{19,20}$

Há evidencias solidas de que a pratica regular de atividade física é capaz de prevenir 
e/ou retardar os danos inerentes ao processo do envelhecimento em idosos eutroficos. ${ }^{21}$ Acredita-se que o estimulo à participação de programas de atividades físicas é capaz de gerar repercussões significativas sobre o estado nutricional e capacidade funcional do idoso. A pratica regular de atividade física é capaz de promover o aumento do ganho de força e massa muscular, tanto através do recrutamento de fibras com a realização de exercícios resistidos, quanto o com estresse metabólico a partir de treinamentos de restrição parcial do fluxo sanguíneo..$^{22,23}$

De maneira geral, a literatura revela que a pratica de atividade física é um componente crítico e de grande impacto para a manutenção da massa corporal magra e da preservação da capacidade funcional do idoso, visto que há deterioração devido ao próprio processo fisiológico que ocorre ao longo da vida. Portanto, o estimulo à prática de atividade física, mesmo em indivíduos longevos com algum grau de déficit nutricional, é capaz de reduzir a morbimortalidade e de retardar déficits cognitivos, nutricionais e funcionais. A atividade física ainda evita a progressão de condições crônicas degenerativas, protela os efeitos inerentes ao envelhecimento e proporciona aumento dos níveis de qualidade de vida e de autopercepção de saúde. 3,18,24,25

Os achados desta pesquisa evidenciaram fatores significativos para a manutenção da funcionalidade de um grupo de idosos com baixo peso assistidos por uma operadora de planos de saúde, do município de São Paulo, SP, Brasil. No entanto, em virtude das particularidades da amostra e do delineamento do estudo, esses resultados não permitem a sua generalização.

\section{CONCLUSÃO}

Conclui-se que o grupo estudado se caracteriza por apresentarassociação de agravos e comportamentos que podem contribuir para desfechos negativos e indesejados. Verificouse ainda que a idade avançada, a dificuldade de mobilidade e os baixos níveis de atividade física se apresentaram como fatores associados ao extremo baixo peso nestes idosos. Além disso, a cada uma hora de atividade física praticada por estes idosos portadores de baixo peso, as chances de progressão para o extremo baixo peso é reduzida em até $14 \%$. Diante dos resultados encontrados para este grupo, fica clara a relevância da prescrição de atividades físicas por profissionais da saúde para indivíduos longevos e, principalmente com algum grau de déficit nutricional como medida de promoção à saúde e prevenção de agravos.

\section{REFERÊNCIAS}

1. Brasil, Ministério da Saúde. Caderno de atenção básica $n^{\circ}$ 19: Envelhecimento e saúde da pessoa idosa. Brasília: Ministério da Saúde; 2006.

2. Pascali C, Peliello LC. Avaliação nutricional. In: Manso MEG (Org.). Geriatria, manual da liga de estudos do processo de envelhecimento. São Paulo: Martinari; 2015. p. 91-104.

3. Sousa KT, Mesquisa LAS, Pereira LA, Azeredo CM. Baixo peso e dependência funcional em idosos institucionalizados de Uberlândia (MG), Brasil. Ciência Saúde Col. 2014;19(8):3513-3520. Doi: https://doi. org/10.1590/1413-81232014198.21472013

4. Sousa VMC, Guariento ME. Avaliação do idoso desnutrido. Rev Bras Clin Med. 2009;7(1):46-9.

5. Organización Panamericana de la Salud (OPAS). Salud Bienestar y Envejecimiento (SABE) en América Latina y el Caribe: informe preliminar. Washington Organización Panamericana de la Salud; c2001 [citado 2019 jan 05]. Disponível em: http://envejecimiento. csic.es/documentos/documentos/paho-salud-01.pdf

6. Martins TI, Meneguci J, Damiâo R. Pontos de corte do índice de massa corporal para classificar o estado nutricional em idosos. RFACS. 2015;3(2):78-87. Doi: https://doi.org/10.18554/refacs.v3i2.10857

7. World Health Organization. Adults 60 year of age and older. In: World Health Organization (WHO). Phsical Status: The use and interpretation of anthropometry. Report of a WHO expert committee. Geneve; WHO; 1995. p. 375-409. [Technical Report Series no. 854]

8. Nutrition Screening Initiative (NSI). Incorporating nutrition screening and interventions into medical practice: a monograph for physicians. Washington, DC: Nutrition Screening Initiative; 1994

9. Manso MEG. Envelhecimento, saúde do idoso e o setor de planos de saúde no Brasil. Rev Kairós - Gerontol. 2017; 20(4):135-51. Doi: https://doi. org/10.23925/2176-901X.2017v20i4p135-151

10. Dias AL, Rodrigues TC. Avaliação da capacidade funcional. In: Manso MEG, editor. Geriatria, Manual da Liga de Estudos do Processo de Envelhecimento. São Paulo: Martinaria; 2015. p. 55-69.

11. Leis DF, Manso MEG. Avaliação de marcha, equilíbrio e atividade física no idoso. In: Manso MEG, editor. Geriatria, Manual da Liga de Estudos do Processo de Envelhecimento. São Paulo: Martinari; 2015. p. 81-89.

12. Serviço de Intervenção nos Comportamentos Aditivos e nas Dependências [homepage on the Internet]. Lisboa: Serviço Nacional de Saúde. c2015 [citado 2019 Jan 05]. Disponível em: http://www. sicad.pt/PT/Intervencao/RedeReferenciacao/ SitePages/Instrumentos.aspx

13. Hernandes ESC, Lebrão ML, Duarte YAO, Santos JLF. Idosos com e sem plano de saúde e características socio epidemiológicas associadas. Rev Saúde Pública 2012;46(6):1030-8. Doi: https://doi.org/10.1590/ S0034-89102012000600013
14. Manso MEG, Osti AV, Borrozino NF, Maresti LTP. Avaliação Multidimensional do Idoso: resultados em um grupo de indivíduos vinculados a uma operadora de planos de saúde. Rev Kairós Gerontol. 2018;21(1):191-211. Doi: https://doi. org/10.23925/2176-901X.2018v21i1p191-211

15. Nunes BP, Batista SRR, Andrade FB, Souza Junior RB, Lima-Costa MF, Facchini LA. Multimorbidade em indivíduos com 50 anos ou mais de idade: ELSI-Brasil. Rev Saude Publica. 2018;52 Supl 2:10s. Doi: https://doi.org/10.11606/s15188787.2018052000637

16. Oliveira HSB, Manso MEG. Tríade iatrogênica em um grupo de mulheres idosas vinculadas a um plano de saúde. Rev Bras Geriatr Gerontol. 2019;22(1):e180188. Doi: https://doi. org/10.1590/1981-22562019022.180188

17. Muniz ECA, Goulart FC, Lazarini CA, Marin MJS. Analise do uso de medicamentos por idosos usuários de plano de saúde suplementar. Rev Bras Geriatr Gerontol. 2017; 20(3):357-87. Doi: https:// doi.org/10.1590/1981-22562017020.160111

18. Ferreira LK, Meireles JFF, Ferreira MEC. Avaliação do estilo e qualidade de vida em idosos: uma revisão de literatura. Rev Bras Geriatr Gerontol. 2018; 21(5): 639-51. Doi: http://dx.doi. org/10.1590/1981-22562018021.180028

19. Tanner RE, Brunker LB, Agergaard J, Barrows KM, Briggs RA, Kwon OS, et al. Age-related differences in lean mass, protein synthesis and skeletal muscle markers of proteolysis after bed rest and exercise rehabilitation. J Physiol. 2015;593(18):4259-73. Doi: http://dx.doi.org/10.1113/JP270699

20. Dutra T, Pinheiro PA, Carneiro JAO, Coqueiro RS, Fernandes $\mathrm{MH}$. Prevalência e fatores associados a sarcopenia em mulheres idosas residentes em comunidade. Rev Bras Cineantropom Desempenho Hum. 2015;17(4):460-71. Doi: https://doi. org/10.5007/1980-0037.2015v17n4p460

21. Gianoudis J, Bailey CA, Daly RM. Associations between sedentary behaviour and body composition, muscle function and sarcopenia in community-dwelling older adults. Osteoporos Int. 2015;26(2):571-9. Doi: https://doi.org/10.1007/ s00198-014-2895-y

22. Viana JU, Dias JMD, Batista PP, Silva SLA, Dias RC Lustosa LP. Effect of a resistance exercise program for sarcopenic elderly women: quasi-experimental study. Fisioter Mov. 2018; 31:e003111. Doi: https://doi.org/10.1590/1980-5918.031.ao11

23. Cardoso RK, Araújo AM, Freitas MP, Rombaldi AJ. Effect of training with partial blood flow restriction in older adults: a systematic review. Rev Bras Cineantropom Hum. 2017; 20(2):21928. Doi: https://doi.org/10.5007/19800037.2018v20n2p219

24. Tecchio JM, Gessinger C. Upper and lower limb functionality and body mass index in physically active older adults. Fisioter Mov. 2017;30(Suppl 1):S45-54. Doi: https://doi.org/10.1590/19805918.030.s01.ao04

25. Malafaia FL, Buglia S. Prescrição de atividade física em idosos: nunca é tarde demais para combatermos o sedentarismo. Rev DERC 2019;25(1):14-8. 